\title{
Functional Insights of MraZ on the Pathogenicity of Staphylococcus aureus
}

\author{
Bingjie Wang ${ }^{1} * *$ \\ Jingjing Duan ${ }^{2, *}$ \\ Ye Jin ${ }^{3, *}$ \\ Qing Zhan ${ }^{4}$ \\ Yanlei $\mathrm{Xu}^{4}$ \\ Huilin Zhao' \\ Xinyi Wang' \\ Lulin $\mathrm{RaO}^{3}$ \\ Yinjuan Guo' \\ Fangyou $\mathrm{Yu}^{1,5}$
}

'Department of Clinical Laboratory, Shanghai Pulmonary Hospital, School of Medicine, Tongji University, Shanghai,

People's Republic of China; ${ }^{2}$ Department of Clinical Laboratory, Renmin Hospital, Hubei University of Medicine, Hubei, People's Republic of China; ${ }^{3}$ Department of Laboratory Medicine, The First Affiliated Hospital of Wenzhou Medical University, Wenzhou, People's Republic of China; ${ }^{4}$ Jiangxi Provincial Key Laboratory of Preventive Medicine, School of Public Health, Nanchang University, Nanchang, People's Republic of China; ${ }^{5}$ Shanghai Key Laboratory of Tuberculosis, Shanghai Pulmonary Hospital, Tongji University School of Medicine, Shanghai, People's Republic of China

*These authors contributed equally to this work
Correspondence: Fangyou Yu; Yinjuan Guo Emailwzjxyfy@163.com; gyjlyy@163.com
Introduction: In recent years, multidrug-resistant methicillin-resistant Staphylococcus aureus has become increasingly prevalent, which raised a huge challenge to antibiotic treatment of infectious diseases. The anti-virulence strategy targeting virulent factors is a promising novel therapy for $S$. aureus infection. The virulence mechanism of $S$. aureus was needed to explore deeply to develop more targets and improve the effectiveness of anti-virulence strategies.

Results: In this study, we found $m r a Z$ was highly conserved in $S$. aureus, and its production is homologous with the MraZ of Escherichia coli, a transcriptional regulator involved in the growth and cell division of $E$. coli. To investigate the function of $m r a Z$ in $S$. aureus, we constructed a MW2 mraZ deletion mutant and its complementary mutant for virulence comparison. Although no remarkable influence on the growth, the mraZ deletion mutant led to significantly reduced resistance to human neutrophils and decreased virulence in Galleria mellonella model as well as mouse skin and soft tissue infection models, indicating its essential contribution to virulence and immune evasion to support the pathogenicity of S. aureus infection. RNA-Seq and quantitative RT-qPCR revealed that MraZ is a multifunctional regulator; it involves in diverse biological processes and can up-regulate the expression of various virulence genes by agr and sarA.

Conclusion: $m r a Z$ plays vital roles in the pathyogenicity of $S$. aureus via regulating many virulence genes. It may be an attractive target for anti-virulence therapy of $S$. aureus.

Keywords: Staphylococcus aureus, virulence, mraZ, agr, sarA

\section{Introduction}

Staphylococcus aureus is a common opportunistic pathogen that usually colonizes in human skin and nasal mucosa, which can cause a variety of infections, ranging from mild skin lesion to severe invasive diseases such as endocarditis, osteomyelitis, pneumonia, and even life-threatening septic shock. ${ }^{1-3}$ Moreover, with the emergence of multidrug-resistant methicillin-resistant $S$. aureus (MRSA) strains in recent years, $S$. aureus infections rise a serious concern in public health. A report from Centers for Disease Control and Prevention from USA showed that the mortality rate caused by MRSA infection was 2.88 per 100000 population per year, which leads to approximately 100 trillion dollars in damage to the global economy. ${ }^{4}$ It is obvious that the problem of antibiotic resistance in bacteria has reached the crisis stage.

A key characteristic in pathogenicity of $S$. aureus that promote its infection is the production of a large array of virulence factors, such as hemolysins, pore-forming leukocidins, enterotoxin, and plasma-coagulase, ${ }^{5-8}$ which allow them to resist 
clearance by the host, to invade and gain access to deeper tissues, and to damage host cells. Currently, most treatments of infectious diseases in clinical are based on the ability of antibacterial agents to kill pathogens quickly. However, the harmful effects of bacterial virulence factors are almost inevitable Furthermore, treatment of infections caused by MRSA strains has become more difficult because of the emergence of multidrug-resistant strains. As conventional antibiotics become less valid, the anti-virulence strategies are drawing increasing attention. One of the advantages of the anti-virulence strategies is that it can specifically block the virulence expression of bacteria without killing or inhibiting the growth of bacteria pathogens, and avoid the selective pressure from antibiotics treatment, which will be less likely lead to the emergence of drug resistance. ${ }^{9,10}$ Therefore, the investigation of more unidentified virulence factors of Staphylococcus aureus and its pathogenic mechanism can provide more directions for the antivirulence strategies of Staphylococcus aureus.

The mraZ gene is highly conserved in many bacterial species. In some Gram-positive bacteria, mraZ is associated with the cell division and cell wall biosynthesis, which plays a vital role in the growth of bacteria. ${ }^{11-13}$ A previous study demonstrated that the overproduction of Escherichia coli MraZ could inhibit cell division and was lethal in rich medium at high induction levels and in minimal medium at low induction levels. ${ }^{11}$ Notably, the MraZ from E. coli and S. aureus shares 36\% sequence identity. However, limited studies have evaluated the role of mraZ gene in S. aureus. The biological functions of the mraZ gene in $S$. aureus still require further investigation.

In this study, to better understand the function of MraZ in $S$. aureus, we constructed the MW2 mraZ deletion mutant and its complementary mutant, and investigated the effects of MraZ on the bacterial growth, the virulence and immune evasion capacity. We found that $\mathrm{MraZ}$ is a multi-functional regulator, especially plays a crucial part in $S$. aureus virulence and immune evasion by regulating the expression of extensive virulence genes.

\section{Materials and Methods}

\section{Bacterial Strains and Culture Conditions}

The bacterial strains and plasmids used in this study are listed in Table 1. Given MW2 is a typical community-acquired MRSA isolate with enhanced virulence, and its genetic background has been well established, it was used for the construction of mraZ knockout and complementation. E. coli
Table I The Bacterial Strains and Plasmids Used in the Present Study

\begin{tabular}{|c|c|c|}
\hline $\begin{array}{l}\text { Bacterial } \\
\text { Strains and } \\
\text { Plasmids }\end{array}$ & Description & Source \\
\hline \multicolumn{3}{|l|}{ Strains } \\
\hline S. aureus MW2 & CA-MRSA strain & $\begin{array}{l}\text { Laboratory } \\
\text { stock }^{\mathrm{a}}\end{array}$ \\
\hline$\Delta m r a Z$ & $\begin{array}{l}\text { mraZ deletion mutant using MW2 } \\
\text { as parental strain }\end{array}$ & This study \\
\hline$\Delta m r a Z-C$ & $\begin{array}{l}\Delta m r a Z \text { mutant complemented } \\
\text { with plasmid pRB473 harboring } \\
\text { mraZ gene }\end{array}$ & This study \\
\hline E.coli DH5 $\alpha$ & Clone host strain & $\begin{array}{l}\text { Laboratory } \\
\text { stock }^{\mathrm{a}}\end{array}$ \\
\hline E. coli DCIOB & $\begin{array}{l}\operatorname{dam}^{+} \Delta \mathrm{dcm}^{-} \Delta \mathrm{hsdRMS} \text { endAI } \\
\text { recAI; clone host strain }\end{array}$ & $\begin{array}{l}\text { Laboratory } \\
\text { stock }^{\mathrm{a}}\end{array}$ \\
\hline \multicolumn{3}{|l|}{ Plasmids } \\
\hline PKORI & $\begin{array}{l}\text { Temperature-sensitive } \\
\text { E. coli }\left(A \mathrm{mp}^{r}\right) \text { Staphylococcus } \\
\text { shuttle }\left(\mathrm{Cm}^{r}\right) \text { vector }\end{array}$ & $\begin{array}{l}\text { Laboratory } \\
\text { stock }^{\mathrm{a}}\end{array}$ \\
\hline pKORI- $\Delta m r a Z$ & Recombinant plasmid & This study \\
\hline pRB473 & Shuttle plamid $\left(\mathrm{Cm}^{r}\right)$ & $\begin{array}{l}\text { Laboratory } \\
\text { stock }^{\mathrm{a}}\end{array}$ \\
\hline pRB473-mraZ & $\begin{array}{l}\text { Shuttle plamid }\left(\mathrm{Cm}^{r}\right) \text {, mraZ gene } \\
\text { with its own promoter was cloned } \\
\text { into plasmid pRB473 }\end{array}$ & This study \\
\hline
\end{tabular}

Note: ${ }^{\text {all }}$ these strains were stored in The First Affiliated Hospital of Wenzhou Medical University.

DH5 $\alpha$ and E. coli DC10B was used for staphylococcal cloning host. E. coli strains were cultured in Luria-Bertani (LB) medium, and Staphylococcus strain were grown at tryptic soy broth (TSB) medium. All strains were shaken in an incubator at $37^{\circ} \mathrm{C}, \sim 220 \mathrm{rpm}$ (adjusted appropriately). The medium was supplemented with an appropriate antibiotic (ampicillin $100 \mathrm{mg} / \mathrm{L}$, chloramphenicol $10 \mathrm{mg} / \mathrm{L}$ and anhydrous tetracycline $50 \mu \mathrm{g} / \mathrm{L}$ ) as needed.

\section{Construction of mraZ Knockout Mutant and Complemented Strain}

A knockout mutant of mraZ was constructed using the temperature-sensitive plasmid pKOR1 based on the homologous recombination. ${ }^{14}$ Briefly, the upstream and downstream fragments of $m r a Z$ were amplified, linked 
by the T4 enzyme, and ligated to the vector pKOR1, resulting in recombinant pKOR1- $\Delta m r a Z$. This recombinant plasmid was consecutively transferred into Escherichia coli DH5a for amplification, into DC10B for bypass the $S$. aureus restriction barriers, and then electrotransfered into MW2. Under the dual pressure of high temperature and antibiotics, $\Delta m r a Z$ was generated by the homologous recombination of the upstream and downstream homologous arm in the pKOR1- $\triangle m r a Z$ with the MW2 genome, as previously described. ${ }^{14}$ The deleted mutant $\triangle m r a Z$ was verified by PCR with DNA sequencing and quantitative reverse transcription-PCR (qRTPCR).

To further verify whether the biological alteration of the deletion mutant was caused by the absence of $\mathrm{mraZ}$, we constructed the complementary mutant strain $\triangle m r a Z-C$ using the shuttle plasmid pRB473. Briefly, the marZ with its promoter regions was amplified and inserted into pRB473. The pRB473-mraZ was transferred into $\triangle m r a Z$ by electroporation. The primers used above are listed in Table 2.

\section{RNA Extraction and qRT-PCR}

Total RNA of $S$. aureus was extracted using RNA extraction kit (Tiangen Biotech, Beijing, China) following the manufacturer's instructions. Briefly, overnight cultures were diluted 1:200 into fresh TSB and incubated at $37^{\circ} \mathrm{C}$. After $9 \mathrm{~h}$, the prepared lysostaphin and lysozyme solution were mixed with bacterium suspension for disrupt the cell walls, and then RNA extracted was followed the kit instructions. A PureLink RNA Mini Kit (Invitrogen, Carlsbad, CA, USA) was used to get purified RNA, and then cDNA was obtained using a PrimeScript RT reagent kit (TaKaRa, Japan). The housekeeping gene $g y r B$ was used as an internal reference gene, and relative quantification of Expression levels were calculated by the $2^{-\Delta \Delta C t}$ method. ${ }^{15}$ The primer pairs used in qRT-PCR are listed in Table 3.

\section{Growth Assay}

S. aureus strains were grown to stationary phase (12h) and then diluted (1:200) in TSB medium. All the cultures were incubated at $37^{\circ} \mathrm{C}$ with shaking at $220 \mathrm{rpm}$. The OD600 value was measured hourly for $24 \mathrm{~h}$ for bacterial growth curves. Meanwhile, the cells were plated in serial dilutions on TSB agar at $1 \mathrm{~h}$ intervals for $24 \mathrm{~h}$, and the colonyforming units (CFU) were counted for viable bacterial count at indicated time points. The assay was performed in duplicate.

\section{Analysis of Pigments Production}

To evaluate pigment production, bacteria were incubated into $3 \mathrm{~mL}$ TSB medium at $37^{\circ} \mathrm{C}$ with shaking. After $24 \mathrm{~h}$, the cultures were centrifuged at $8000 \mathrm{rpm}$ for $10 \mathrm{~min}$, and pigment production was assessed visually by the color of centrifugated precipitation.

\section{Hemolytic Capacity}

The hemolytic capacity of $S$. aureus isolates was evaluated based on the zone of clearing around colonies plated on sheep blood agar plates. Briefly, S. aureus strains were cultivated to stationary phase (12h), the cells were collected, washed twice with PBS, and adjusted to OD600 $=1$;

Table 2 Primers Used for Construction of $\Delta m r a z$ and $\Delta m r a z-C$

\begin{tabular}{|c|c|c|}
\hline Primer & Primer Sequence $\left(5^{\prime}-3^{\prime}\right)$ & Underlined \\
\hline mraZ-F & ATGTTCATGGGAGAATACGATCATC & \\
\hline mraZ-R & TAAAAATCAAAATCTATTAAATCTT & \\
\hline mraZ UP-F & $\begin{array}{l}\text { GGGGACCACTTTGTACAAGAAAGCTGGGT } \\
\text { GCTTGAATGCGATGAT }\end{array}$ & attB2 \\
\hline mraZ UP-R & GGAATTCCCACCTCACCTTATATATAATTTAACC & EcoRI \\
\hline mraZ DOWN-F & GGAATTCCGGAGGAATTGAAGTGTTTCATCATA & EcoRI \\
\hline mraZ DOWN-R & $\begin{array}{l}\text { GGGGACAAGTTTGTACAAAAAAGCAGGCT } \\
\text { GCTTCGTGCTCT }\end{array}$ & attBI \\
\hline mraZ HF-F & CGGAATTCCGAATGGGAGGATTACAAGA & ECORI \\
\hline$m r a Z$ HF-R & GGGGTACCCCCCACCTAGCGTACAGTCA & KPNI \\
\hline
\end{tabular}


Table 3 Primers Used in qRT-PCR

\begin{tabular}{|l|l|}
\hline Primer & Primer Sequence (5' ${ }^{\prime} \mathbf{3}^{\prime}$ ) \\
\hline gyrB-RT-F & ACATTACAGCAGCGTATTAG \\
\hline gyrB-RT-R & CTCATAGTGATAGGAGTCTTCT \\
\hline mraZ-RT-F & TGATTATACCGTCCAAGT \\
\hline mraZ-RT-R & CACCAGAGAAGAACATAC \\
\hline sarA-RT-F & AAACCCTGAATTTGAATG \\
\hline sarA-RT-R & GATATTACATCTGCTCCT \\
\hline agrA-RT-F & TCCAGCAGAATTAAGAACTCG \\
\hline agrA-RT-R & ATATCATCATATTGAACATACACT \\
\hline RNAIll-RT-F & CATGGTTATTAAGTTGGGATGGC \\
\hline RNAIII-RT-R & GAAGGAGTGATTTCAATGGCACA \\
\hline hId -RT-F & GAAGGAGTGATTTCAATG \\
\hline hld -RT-R & TGTCGATAATCCATTTTAC \\
\hline sec4-RT-F & TCAGCAACTAAAGTTAAG \\
\hline sec4-TR-R & GCAGTTTACATAGTAATTTG \\
\hline
\end{tabular}

then 10ul each culture suspension was spotted on sheep blood agar plates. The zones of hemolysis (diameter) were measured after $24 \mathrm{~h}$ of $37^{\circ} \mathrm{C}$ incubation. The experiments were conducted in triplicate.

\section{Neutrophil Killing Assays}

Neutrophils with $>95 \%$ purity and viability were isolated from the venous blood of healthy volunteers using the Human Neutrophil Isolation Kit (Haoyang, Tianjin, China). In brief, $S$. aureus isolates were grown to midlogarithmic growth phase, collected, washed, and resuspended in PBS. After the opsonization of $5 \%$ normal human serum, $1.0 \times 10^{5} \mathrm{CFU}$ bacterial suspension were mixed with $1.0 \times 10^{6}$ neutrophils in RPMI 1640 medium at $37^{\circ} \mathrm{C}$ with gentle rotation. Samples were taken in $1 \mathrm{~h}, 2 \mathrm{~h}$, and $3 \mathrm{~h}$ for CFU enumeration. $0.25 \%$ TritonX-100 was added to each sample to ensure lysis of phagosomes before diluting and plating on TSB agar. The experiments were done in triplicate.

\section{Galleria mellonella Infection Model}

As previously described, larvae of G. mellonella weighing 250-350mg (Tianjin huiyude Biotech, Tianjin, China) were stored in the dark at $10^{\circ} \mathrm{C}$ and used within a week of delivery. Overnight cultures of $S$. aureus were collected, washed, and resuspended in PBS. G. mellonella was injected with $3.0 \times 10^{6} \mathrm{CFU} /$ larvae, via the last right proleg using Hamilton syringe. Each experimental group contained 10 larvae. Larvae with injected sterile PBS and uninjected larvae as control. The treated larvae were placed in clean, sterile plastic petri dish and incubated at $37^{\circ} \mathrm{C}$. The survival rate was monitored every $12 \mathrm{~h}$ for 3 days. The results were analyzed by GraphPad Prism 6 software. The experiment was repeated three times with similar results.

\section{Mouse Model of Skin Abscess}

The mouse skin infection model was performed as described previously. Female BALB/C nude mice aged 4 to 6 weeks old and weighing about $22 \mathrm{~g}$ were used for the experiments. In brief, all mice were administered with sterile feed, pure water one week before the time of use to adapt to the experimental environment. $S$. aureus strains were grown to mid-logarithmic phase as described above. The mice were injected with $100 \mu \mathrm{L}$ PBS containing $1.0 \times 10^{7} \mathrm{CFU}$ S. aureus, and mice injected with $100 \mu \mathrm{L}$ PBS as control. Each experimental group contained 10 mice. Abscess formations were observed and recorded daily for 7 days. The length (L) and width (W) were measured to calculate the size of the abscesses follow the formula: $A=\pi(L \times W) / 2$. All animals were sacrificed 7 days post infection.

\section{Cytokine Expression in Bacteremia Mouse Model}

BALB/C nude mice were immunodeficient animals with reduced cellular immunity and humoral immune function, which might affect the production of inflammatory cytokines. Therefore, BALB/C female mice with complete immunity capacity aged 4 to 6 weeks old were used for the bacteremia model and were tested for the expression levels of cytokine. Briefly, mice were randomly divided into four groups, and each group contains 10 mice. The mice were infected by intraperitoneal injection with 100ul PBS containing $1.0 \times 10^{7}$ CFU S. aureus, and mice injected with $100 \mu \mathrm{L}$ PBS as control. They were raised under the same conditions for $3 \mathrm{~L}$ days, and then 7 mice were randomly selected for further analysis (exclude the death mice). Blood samples were obtained, and the level of cytokines in the sera of each mouse was detected by ELISA method. 


\section{RNA-Sequencing and Data Analysis}

$S$. aureus strains were inoculated for $9 \mathrm{~h}$ in TSB medium at $37^{\circ} \mathrm{C}$, and total RNA was extracted as above. Qualitative RNA samples were analyzed by RNA-seq (transcriptome sequencing) using an Illumina HiSeq $\mathrm{X}$ platform and the pe150 (150 bp double-stranded assay) strategy. Differential expression analysis was performed by DEGseq software on the RNA sequencing reads. Gene was considered significantly differentially regulated with $\log 2$ (fold change) $\mid>1$ and $P<0.005$.

\section{Statistical Analysis}

Statistical comparisons were performed using SPSS (version 16.0) and GraphPad Prism 8. Two groups comparison was analyzed using unpaired $t$-test. Three or more groups comparison was analyzed using one-way or two-way ANOVA. The survival curve was analyzed by the Log rank test (Mantel-Cox). The threshold for statistical significance adopted in all analyzes was $P<0.05$.

\section{Ethics Statement}

All procedures involving human participants were performed in accordance with the ethical standards of First Affiliated Hospital of Wenzhou Medical University. All volunteers gave written informed consent in accordance with the Declaration of Helsinki prior to donating blood. All animal assays were carried out in accordance with The Regulation on the Management of Laboratory Animals for the welfare of the laboratory animals, and the protocol was approved by the Institutional Animal Care and Ethics Committee at First Affiliated Hospital of Wenzhou Medical University.

\section{Results}

\section{Construction of mraZ Deletion Mutant Strains}

In the genome of $S$. aureus MW2 (GeneBank accession number: GCF_000011265.1), mraZ is a 432bp gene encoding a 143 amino acid protein with contained SpoVT-AbrB DNA-binding domains, and it is located downstream of the putative cysteine ligase encoding gene $b s h C$ and upstream of the 16S rRNA methyltransferases encoding gene $r s m H$.

The temperature-sensitive shuttle plasmid pKOR1 was used to generate the $\triangle m r a Z$ mutant in the MW2 strain. The complementary strain of $\triangle m r a Z$ mutant named $\triangle m r a Z-C$ was constructed using the vectors pRB473. All mutants were verified by PCR with sequencing and qRT-PCR. As shown in Figure 1, the $\Delta m r a Z$ mutant and $\Delta m r a Z-C$ were successfully constructed.

\section{MraZ Has No Effect on the Growth and Pigment Production, While Enhances the Hemolysis Capacity of S. aureus}

In order to investigate the potential impact of the mraZ deletion on $S$. aureus growth rate, the growth curves and the viable bacterial counts of strains MW2, $\Delta m r a Z$ and $\triangle m r a Z-C$ were determined. Compared with MW2 and $\triangle m r a Z-C$, no substantial differences in the growth curve or the viable bacterial count were found in $\triangle m r a Z$, indicating that the absence of mraZ gene did not affect the growth of $S$. aureus (Figure 2A and B). We also found that there is no difference in pigment production between $\triangle m r a Z$ and MW2 or $\triangle m r a Z$-C (Figure 2C). We further examined the hemolysis ability of the bacteria, one of the most important virulence phenotypes of $S$. aureus, and found that the hemolytic capability of $\triangle m r a Z$ was significantly reduced (Figure 2D).

\section{MraZ Makes a Significant Contribution to Virulence of S. aureus in the G. mellonella Infection Model}

To investigate whether the absence of $m r a Z$ can lead to less virulence, we first compared the survival rates of the wide-type strain MW2, $\triangle m r a Z$ deletion mutant and $\Delta$ mraZ-C in G. mellonella infection model. As shown in Figure 3, the survival rate of G. mellonella infected with $\Delta m r a Z$ deletion mutant was significantly higher than the

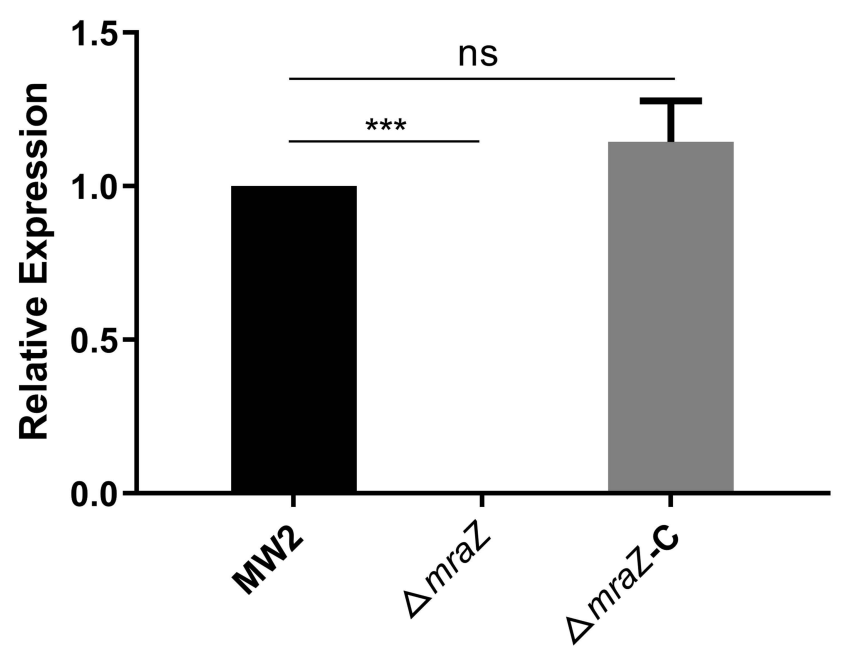

Figure I Validation of the mraZ expression level of the knockout strain and complementary strain. The mraZ expression level of the wild strain used as a control, was regarded as I. $* * * p<0.00 \mathrm{I}$; ns, $p \geq 0.05$. 

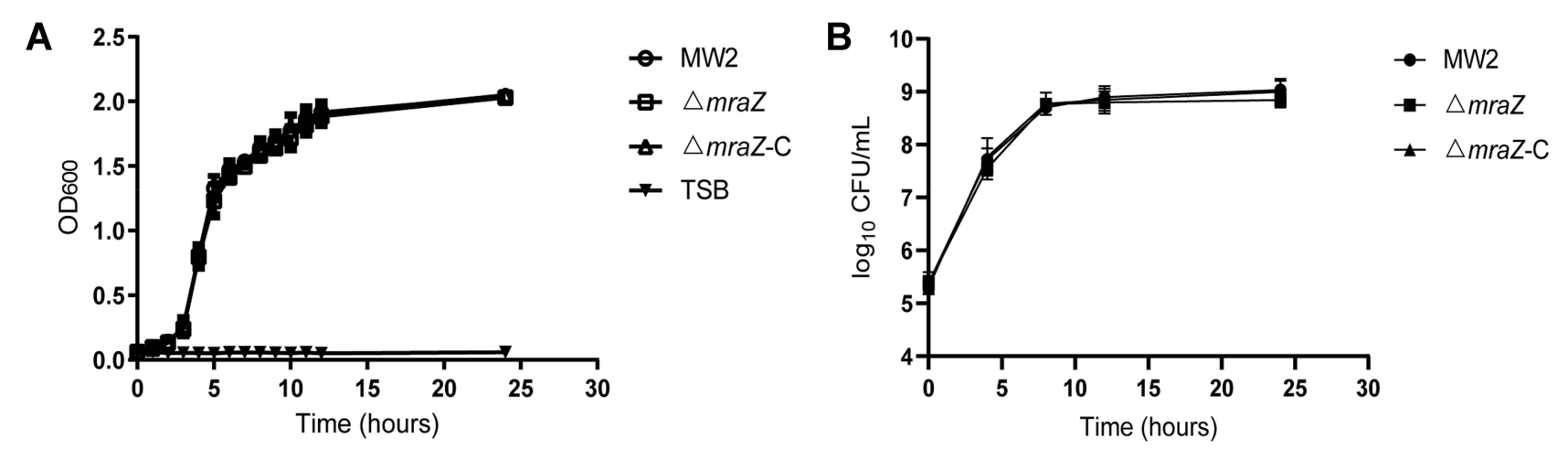

C

D


Figure 2 The role of MraZ in S. aureus MW2, $\triangle m r a Z$ and $\triangle m r a Z-C$ phenotypes. (A) The growth curve by measuring the $\mathrm{OD}_{600}$. (B) Viable bacteria count by measuring CFU on TSB agar plates. (C) Production of pigments by MW2, $\triangle m r a Z$ and $\triangle m r a Z-C$. (D) Analysis of the hemolysis capacity by the zone of Hemolysis on sheep blood agar plates. ${ }^{* *} p<0.01$.

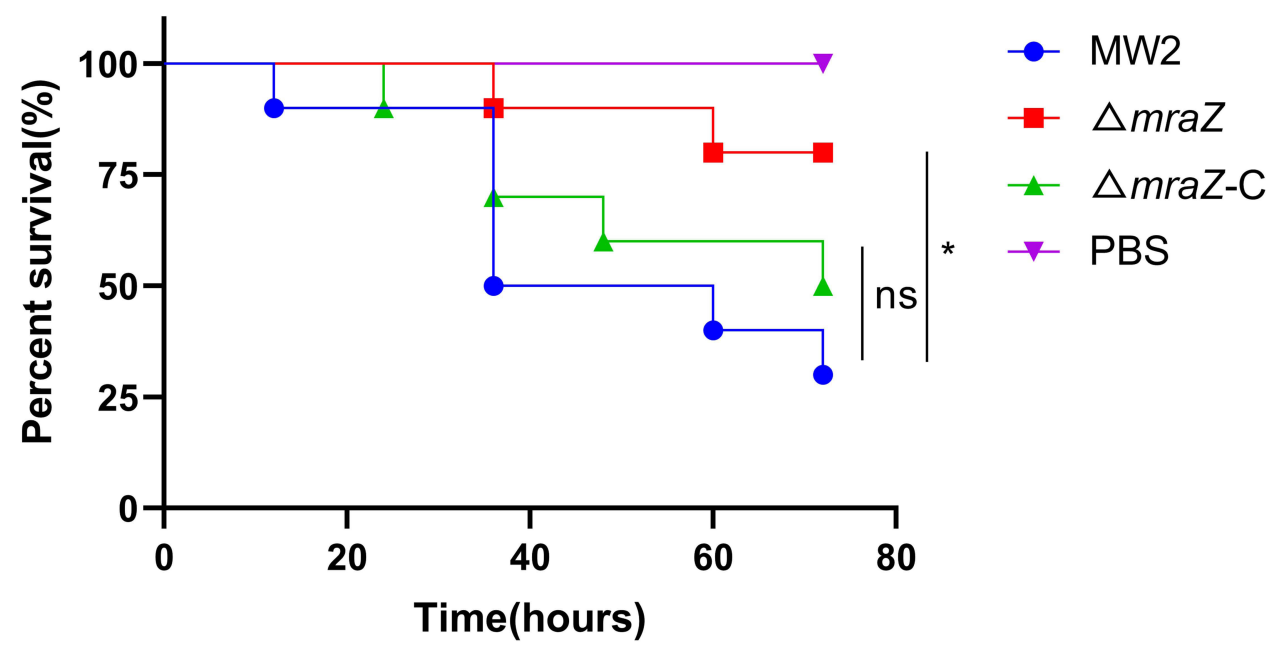

Figure 3 Galleria mellonella infection model. The survival rates of the $G$. mellonella after infection with MW2, $\Delta m r a Z$ and $\Delta m r a Z-C$ were expressed by the Kaplan-Meier survival plot. $* p<0.05 ;$ ns $p \geq 0.05$. 
wide-type strain, while the virulence of $\Delta m r a Z-C$ was restored to the wild-type level.

\section{MraZ of S. aureus Promotes the Formation of Skin Abscesses in Mouse}

To further verify the role of $m r a Z$ in the invasiveness and acute virulence potential of $S$. aureus, we established mouse models of skin abscess infection against MW2, $\Delta m r a Z$ and $\triangle m r a Z-C$ and compared their ability to cause pathological lesions or abscesses. As show in Figure 4, all the mice infected with these three stains were found skin lesion; however, the abscess area of the $\triangle m r a Z$ was significantly smaller than the MW2, indicating that MraZ plays a considerable role in the process of abscess formation.

\section{MraZ of S. aureus Helps Evade the Elimination of Human Neutrophils}

To determine the role of $m r a Z$ in $S$. aureus immune evasion, we evaluated the survival rates of the wide-type strain MW2, $\Delta m r a Z$ and $\Delta m r a Z-C$ in human neutrophils. As shown in Figure 5, the survival rate of $\triangle m r a Z$ was lower than the parent strain after $1 \mathrm{~h}$ of incubation $(p<$ $0.05)$. After incubated for $2 \mathrm{~h}$, a significant reduction of the survival rate of $\triangle m r a Z$ deletion mutant was found compared with the wide-type strain MW2. It indicated that the presence of mraZ gene could help resist clearance by the host innate immune system, thereby likely contributing to the promotion of virulence.

\section{The mraZ Deletion Mutant Has Lower Pro-Inflammatory Cytokine Expression Levels in Mouse Bacteremia Model}

Cytokine play major roles in the innate immune system by the interaction of pro-inflammatory cytokines such as TNF- $\alpha$ and IL-6 and anti-inflammatory cytokines such as IL-10 and IL-4. Therefore, we injected the bacteria into abdominal cavity of mice and determined the cytokines levels of MW2, $\Delta m r a Z$ and $\triangle m r a Z-C$ to evaluate the role of $m r a Z$ in the inflammatory response in $S$. aureus infection. As shown in Figure 6, the levels of TNF- $\alpha$ and IL-6 in $\Delta m r a Z$ were both significantly lower than the other two strains $(p<$ 0.01 ), while IL-4 and IL-10 have no significant difference in these strains, suggesting that the presence of mraZ can stimulate the production of pro-inflammatory cytokines and promote the inflammatory response to $S$. aureus.
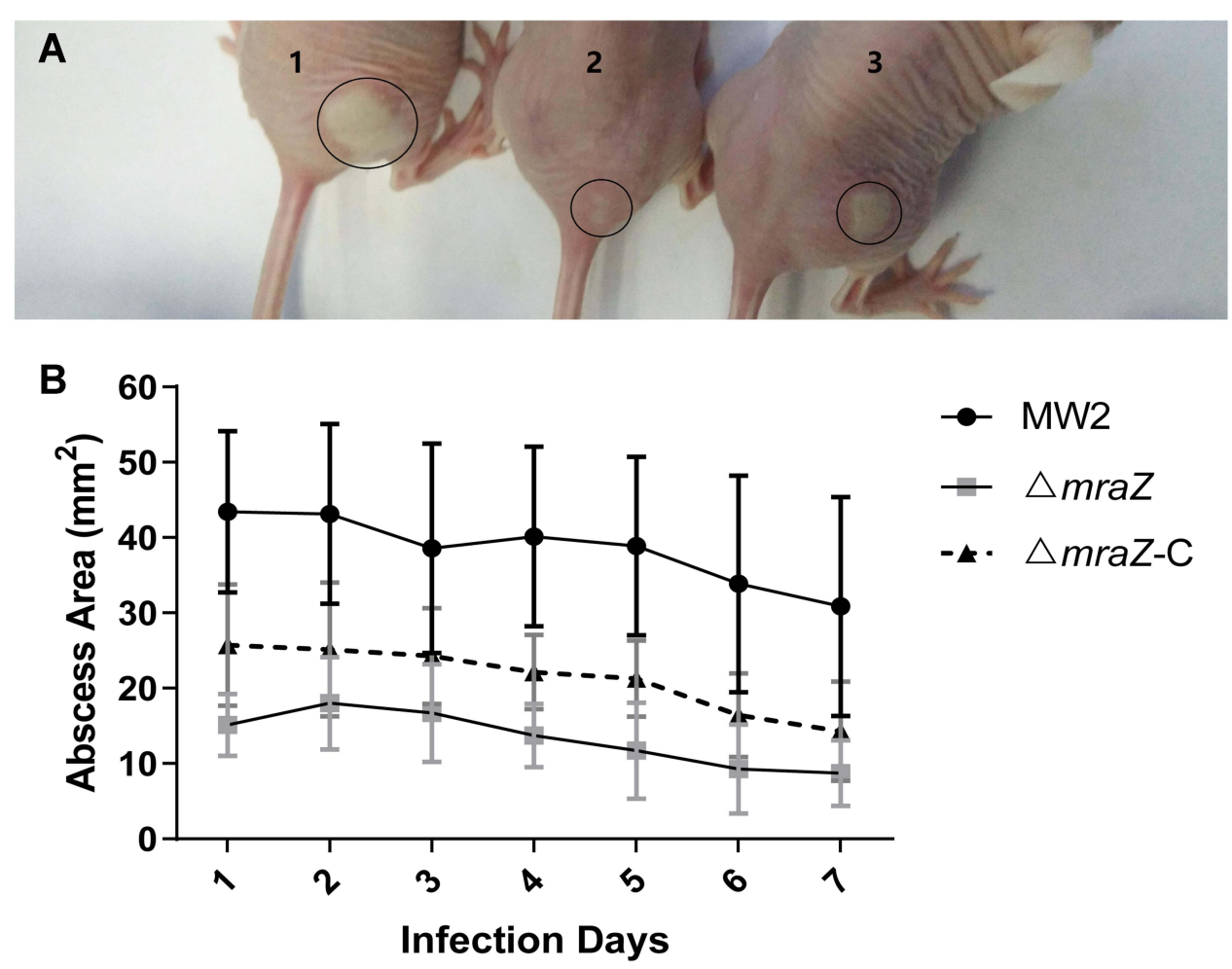

Figure 4 Comparison of infected area of mouse skin abscess. (A) The picture of representative abscesses on days 2 after infection. The No. I indicated an abscess after infection with a wild-type MW2 strain, No. 2 was a $\Delta m r a Z$ strain, and No. 3 was a $\Delta m r a Z-C$ strain. (B) a plot of the area changes of abscesses during skin infections in mice every day. 


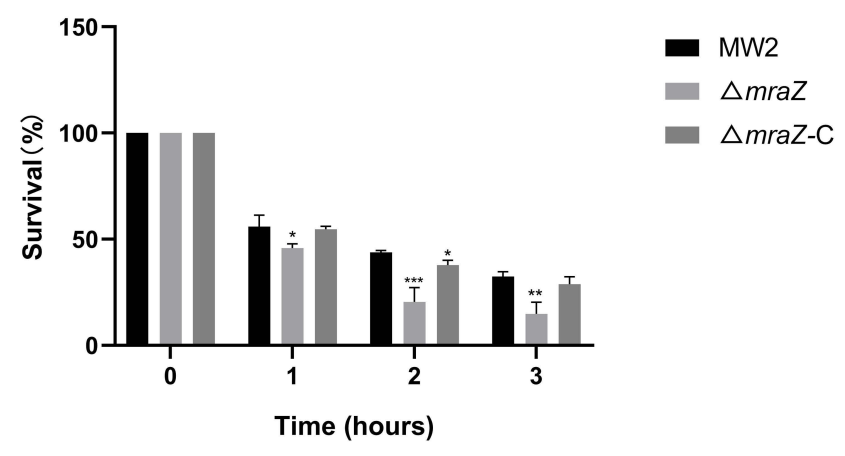

Figure 5 Survival rates of $S$. aureus $M W 2, \Delta m r a Z$ and $\triangle m r a Z-C$ after incubated with human neutrophils. ${ }^{*} p<0.05 ; * * p<0.0$ I; ${ }^{* * *} p<0.00$ I.

\section{Transcriptome Comparison of MW2 and} $\Delta$ mraz

With the involvement of the $\operatorname{mar} Z$ gene in virulence and immune evasion of $S$. aureus, we speculated MraZ might involve in regulating the expression of various genes. Therefore, we performed RNA-Seq analysis on MW2 and MW2 $\Delta m r a Z$. Compared with the wild strain of MW2, there were 335 genes in the $\Delta m r a Z$ mutant strain with differential expression $\geq 2$-fold, of which 187 were up-regulated and 148 were down-regulated (Figure 7A). Furthermore, The Go enrichment analysis revealed that the differentially expressed genes (DEGs) are related to multiple functions, mainly the decomposition and anabolism of multiple substances, DNA replication and repair, gene expression regulation, ribosome synthesis, material transport, cell division and infection (Figure 7B). Therefore, MraZ can act as a multi-functional regulator and affect the expression of various genes.

In the mraZ deletion mutant, the transcription levels of the two-component system agr was down-regulated 8.51fold ( $2^{3.09}$-fold). The expression of $d l t$, an operon mediates d-alanine incorporation into teichoic acid and

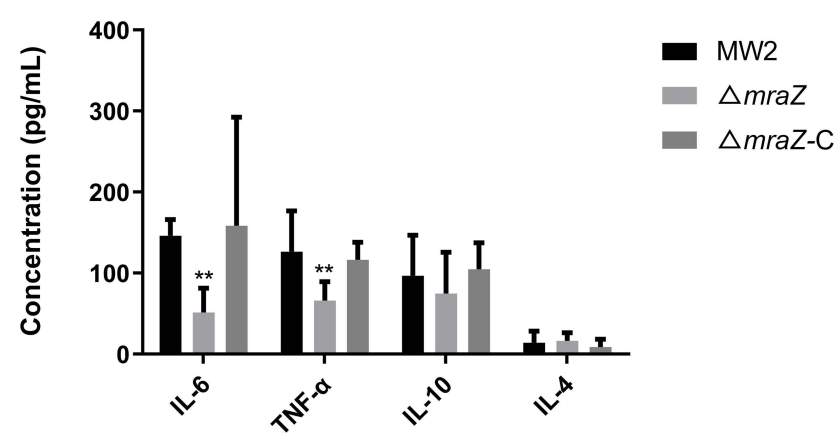

Figure 6 Comparison of Cytokine expression levels in mice after infection with MW2, $\Delta m r a Z$ and $\triangle m r a Z-C$, respectively. $* * p<0.01$. a determinant of defensin resistance that is essential for resistance to human neutrophil killing and virulence in mice, ${ }^{16}$ was down-regulated 2.58 -fold $\left(2^{1.37}\right.$-fold $)$ in MW2 $\Delta m r a Z$. In addition, the transcription of clfA encoding fibrinogen-binding MSCRAMM clumping factors A was significantly down-regulated 2.16 -fold (2.11 fold), which might associated with the decreased virulence of S. aureus $^{17}$ (Table 4). Although the expression of Staphylokinase encoding gene $s a k$, superantigen-like protein encoding gene ssll3 were up-regulated, the transcription of the beta-class phenol-soluble modulin (PSM) encoding gene $p \sin \beta$, alpha family PSM encoding gene psma 3 and hld, Alpha-hemolysin encoding gene hly/hla, and enterotoxin type $\mathrm{C}$ encoding gene sec 4 were downregulated 61.82-fold ( $2^{5.95}$ fold), 50.21-fold ( $2^{5.65}$ fold), 33.13 -fold ( $2^{5.05}$ fold), 2.13-fold ( $2^{1.09}$ fold) and 3.12fold ( $2^{1.64}$ fold), respectively (Table 4$)$. These data suggested that the decreased virulence and compromised immune phenotype resulted from the absence of mraZ was associated with the down-regulated expression of agr, dlt, clfA, psma, psm $\beta$, hly/hla, hld and sec4.

\section{The Transcription Levels of Well-}

\section{Established Virulence Factors and} Regulatory Genes in the MW2, $\Delta m r a z$ and $\triangle m r a z-C$

According to the absence of mraZ contributed to the virulence and immune evasion phenotype, we next measured the expression levels of several well-established virulence factors and regulator genes in detail in the MW2, $\Delta m r a Z$ and $\triangle m r a Z-C$ by qRT-PCR. As shown in Figure 8 , the expression of hld and $\sec 4$ were significantly down-regulated in $\triangle m r a Z$ strain $(p<0.05)$; hemolysin and enterotoxin encoded by these two genes were the topical exotoxins contributed to the virulence of $S$. arueus, conforming that the $m r a Z$ affected the expression of virulence factors such as hemolysin or enterotoxin in S. aureus. The $a g r$ system consists of two adjacent transcripts RNA II and RNA III. RNA III can drive expression of most $a g r$ dependent virulence factors, such as the Alpha-hemolysin and delta-hemolysin, and agrA can promote the transcription of PSM genes. ${ }^{18}$ In agreement with the transcriptome data, the expression level of the agrA and RNAIII in $\triangle m r a Z$ were significantly lower than the MW2 strain $(p<0.05)$. Furthermore, we also investigated the expression of the major $S$. aureus virulence regulatory locus sarA under the same conditions. We found that the expression 
A

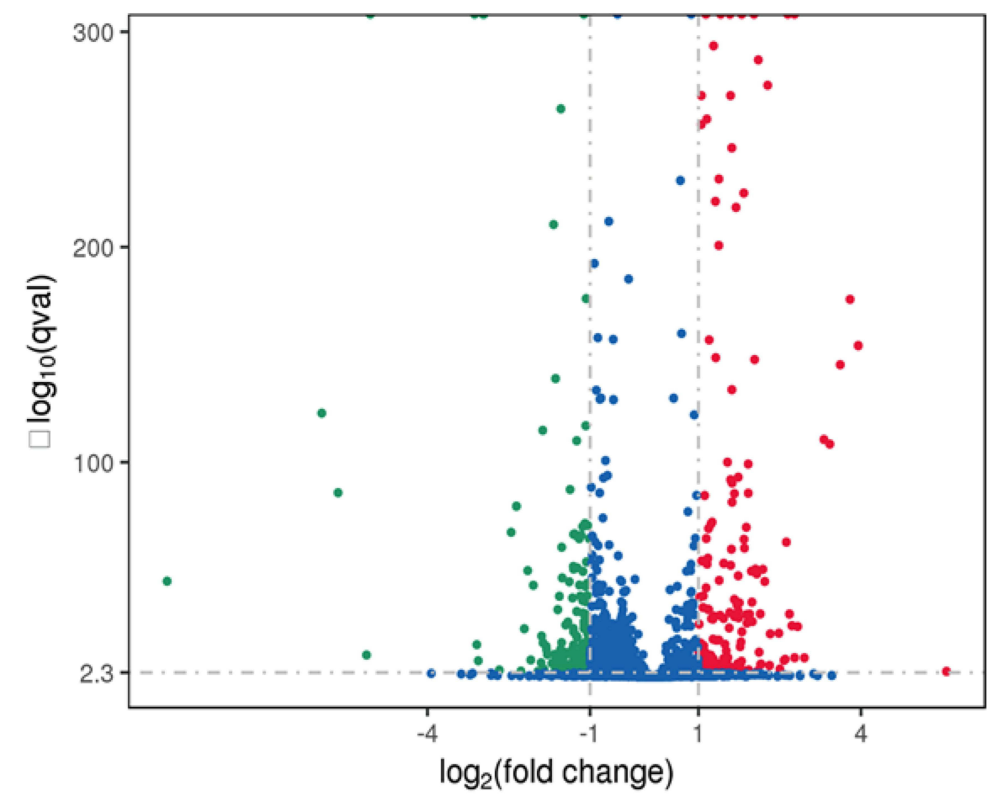

DEGs ( 335 )

- up: 187

- down: 148

B

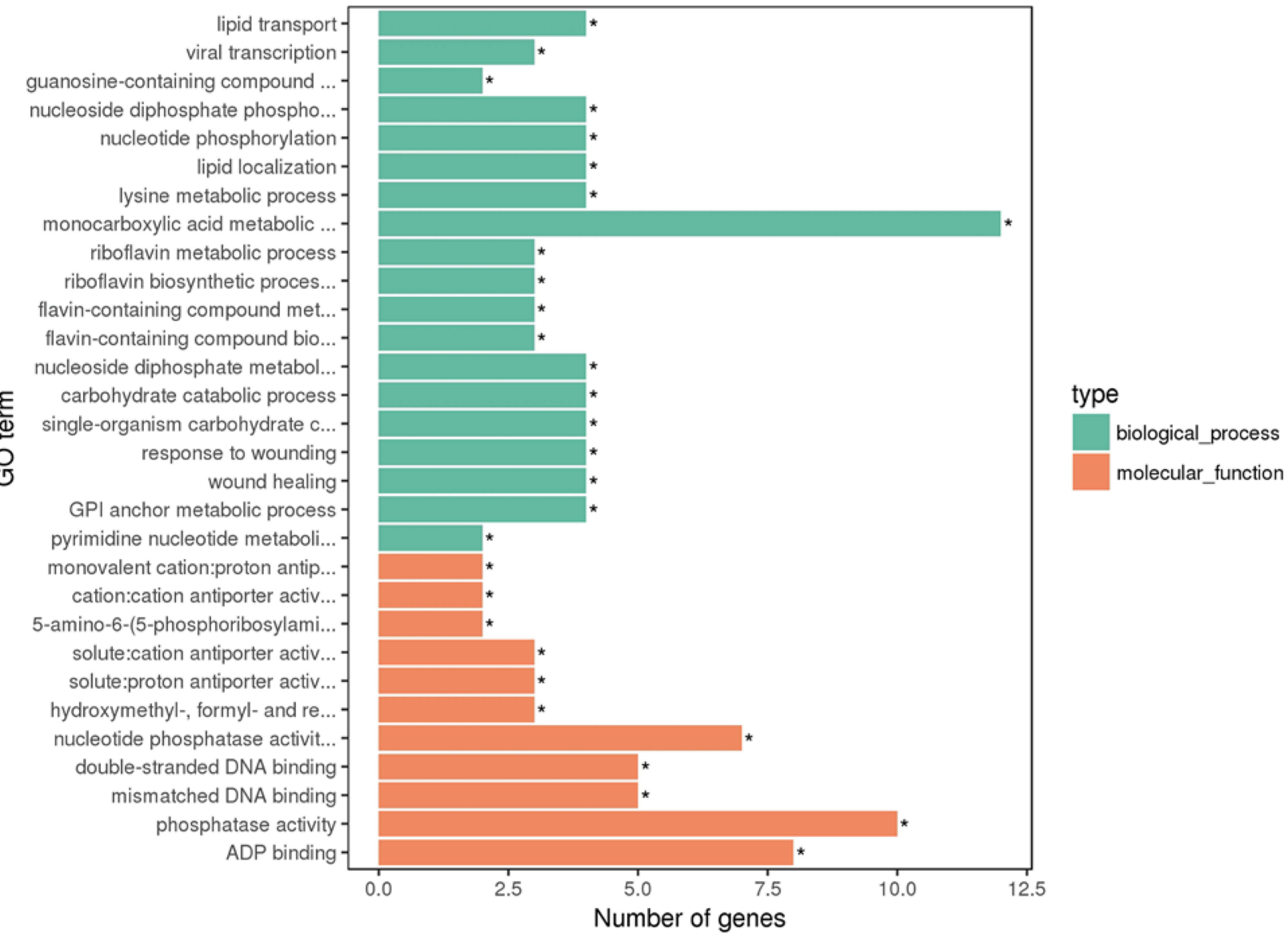

Figure 7 (A) Volcano plot of differences in gene expression between $\Delta m r a Z$ and MW2. The abscissa refers to the fold-change in the two samples; the ordinate refers to the statistically significant difference in gene expression; red dots indicate a significant difference in up-regulated genes and green dots indicate down-regulated genes. A gene with $\mid \log 2$ (Fold Change) $\mid>I$ and q value $<0.005$ between MW2 and $\Delta m r a Z$ was considered to be significantly differentially expressed. (B) $\Delta$ mraZ vs MW2 differential gene GO enrichment histogram. The ordinate is the enriched GO term, and the abscissa is the number of differential genes in the term. Different colors are used to distinguish biological process and molecular function, with *For enriched GO term. 
Table 4 Gene Expression Changes Associated with Virulence or Immune Evasion

\begin{tabular}{|c|c|c|c|c|}
\hline Gene_ID & log2.Fold & p value & q value & Description \\
\hline \multicolumn{5}{|c|}{ Two-component system } \\
\hline MW_RS03395 & -1.1112 & $6.42 \mathrm{E}-26$ & $2.8 \mathrm{IE}-25$ & Bacitracin $A B C$ transporter permease $V r a G$ \\
\hline MW_RSI0660 & -2.9599 & 0 & 0 & Accessory gene regulator AgrB \\
\hline MW_RSI0665 & -3.0897 & $1.94 \mathrm{E}-16$ & $6.07 E-16$ & Accessory gene regulator AgrD \\
\hline MW_RS03390 & -1.3327 & $5.76 \mathrm{E}-14$ & $1.60 \mathrm{E}-13$ & $\mathrm{ABC}$ transporter ATP-binding protein $\mathrm{VraF}$ \\
\hline MW_RSI0675 & -2.9722 & 0 & 0 & Accessory gene regulator AgrA \\
\hline \multicolumn{5}{|c|}{ Staphylococcus aureus infection } \\
\hline MW_RS03395 & -1.1112 & $6.42 \mathrm{E}-26$ & $2.8 \mathrm{IE}-25$ & Bacitracin $A B C$ transporter permease $V r a G$ \\
\hline MW_RS044I5 & -1.3686 & $3.27 \mathrm{E}-89$ & $4.98 \mathrm{E}-88$ & D-alanyl-lipoteichoic acid biosynthesis protein DItB \\
\hline MW_RS04425 & -1.1376 & $6.26 \mathrm{E}-72$ & 7.8IE-7I & D-alanyl-lipoteichoic acid biosynthesis protein DItD \\
\hline MW_RS044I0 & -1.2454 & $5.98 \mathrm{E}-\mathrm{I} 12$ & I.09E-II0 & D-alanine-poly(phosphoribitol) ligase subunit DltA \\
\hline MW_RS03390 & -1.3327 & $5.76 \mathrm{E}-14$ & $1.60 \mathrm{E}-13$ & $\mathrm{ABC}$ transporter ATP-binding protein $\mathrm{VraF}$ \\
\hline MW_RS04I55 & -1.1143 & 0 & 0 & MSCRAMM family adhesin clumping factor ClfA \\
\hline MW_RS04I 25 & -1.6362 & $5.94 \mathrm{E}-|4|$ & $1.38 \mathrm{E}-139$ & Staphylococcal enterotoxin type $\mathrm{C} \mathrm{SeC4}$ \\
\hline MW_RSI0280 & 1.7703 & I.06E-06 & $1.68 \mathrm{E}-06$ & Staphylokinase Sak \\
\hline \multicolumn{5}{|l|}{ Cell killing } \\
\hline MW_RS05625 & -1.0928 & $2.14 \mathrm{E}-73$ & 2.77E-72 & Alpha-hemolysin \\
\hline MW_RSI0655 & -5.0549 & 0 & 0 & Delta-hemolysin \\
\hline \multicolumn{5}{|c|}{ Toxin biosynthetic process } \\
\hline MW_RS02670 & -1.0167 & $3.37 E-19$ & $1.20 \mathrm{E}-18$ & PIN/TRAM domain-containing protein \\
\hline \multicolumn{5}{|l|}{ Pathogenesis } \\
\hline MW_RSI0280 & 1.7703 & $1.06 \mathrm{E}-06$ & $1.68 \mathrm{E}-06$ & Staphylokinase Sak \\
\hline MW_RS05645 & 1.8343 & 0.0022839 & 0.0024122 & Superantigen-like protein SSLI3 \\
\hline MW_RS04I25 & -1.6362 & $5.94 \mathrm{E}-\mathrm{I} 4 \mathrm{I}$ & $1.38 \mathrm{E}-139$ & Staphylococcal enterotoxin type $\mathrm{C} 2 \mathrm{SeC} 4$ \\
\hline MW_RS05690 & -5.9501 & $7.8 \mathrm{IE}-125$ & $1.57 \mathrm{E}-123$ & Beta-class phenol-soluble modulin betal \\
\hline MW_RSI0660 & -2.9599 & 0 & 0 & Accessory gene regulator AgrB \\
\hline MW_RS05695 & -5.1239 & $1.69 \mathrm{E}-1 \mathrm{I}$ & $3.99 \mathrm{E}-\mathrm{II}$ & Beta-class phenol-soluble modulin beta2 \\
\hline MW_RSI4485 & -5.6492 & $1.02 \mathrm{E}-87$ & $1.53 \mathrm{E}-86$ & Alpha-3 family phenol-soluble modulin \\
\hline \multicolumn{5}{|l|}{ Cell adhesion } \\
\hline MW_RSI3825 & -2.2759 & 0.0010466 & 0.0011656 & Hypothetical protein \\
\hline MW_RS04I55 & -1.1143 & 0 & 0 & MSCRAMM family adhesin clumping factor ClfA \\
\hline
\end{tabular}

level of sarA in $\triangle m r a Z$ also significantly decreased $(p<0.05)$. Additionally, except sec4, the expression of most above measured virulence genes and regulatory genes in the MW2 $\Delta$ mraZ-C were approximately recovered to the wild-type strain MW2 (Figure 8). Therefore, it is likely that the $m r a Z$ can activate the expression of the virulence factors by up regulating several key regulatory genes such as agr and $\operatorname{sar} A$.

\section{Discussion}

In recent years, the anti-virulence strategies targeting multiple virulence factors of $S$. aureus have been proved to be an effective alternatives to antibacterial drugs in the treatment of bacterial infection; it is reported that clonal antibody against a-hemolysin could serve as an effective target for monoprophylaxis or adjuvant therapy for S. aureus pneumoniae; ${ }^{19}$ Diapophytoene desaturase CrtN, 


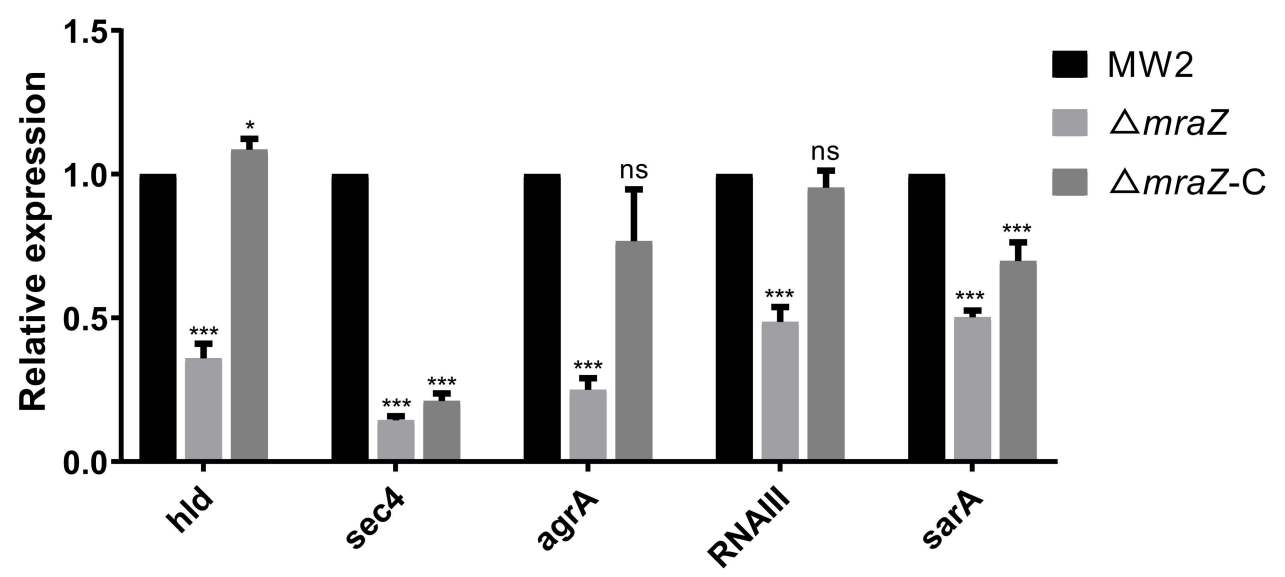

Figure 8 Relative transcription of various genes in MW2, $\triangle m r a Z$ and $\triangle m r a Z-C$ detected by RT-qPCR. Data are mean \pm SD of triplicate experiments and analyzed by oneway analysis of variance (ANOVA) with Dunnett's multiple comparison test compared with the wide-type strain MW2. $* p<0.05$; $* * * p<0.00 \mathrm{I}$.

a key catalytic enzyme of the golden pigment biosynthesis pathway, can also effectively block the virulence of bacteria by targeting drugs. $^{20}$ However, S. aureus strains usually contain various virulence genes and display heterogeneous virulence profiles. In this study, for the first time, we revealed that MraZ highly conserved in $S$. aureus is a multi-functional regulator, and elucidated that it plays an essential role in the virulence and the innate host defense of $S$. aureus. Our research suggested a promising target for anti-virulence strategy for $S$. aureus infection.

Studies involving mraZ in E. coli, Corynebacterium glutamicum and other Gram-negative bacteria have described its position at the head of the division cell wall $(d c w)$ operon (mraZ-mraW-ftsL-ftsI-murE-murF-mraYmurD-ftsW-murG-murC-fts $Q$-ftsA-ftsZ-envA), which responsible for cell division and cell wall synthesis. $\mathrm{MraZ}$ can as regulator to control the expression of the first 11 genes of $d c w$ operon and excessive production of MraZ inhibits cell division in E. coli. ${ }^{11-13}$ We found $m r a Z$ was highly conserved in $S$. aureus, while it is located downstream of the $b s h C$ gene and upstream of the $r s m H$ gene, which is different from other reported Gramnegative bacteria. Unsurprisingly, in our study, the MraZ in $S$. aureus have no effect on the bacterial growth, indicating the species-specific functions of MraZ homologs in bacteria.

We found that the MraZ protein of $S$. aureus MW2 contains SpoVT-AbrB DNA-binding domains, which existed in some toxin-antitoxin system of bacteria. ${ }^{21,22}$ Moreover, the downstream gene $r s m H$ was proved to be related to the virulence of $S$. aureus in the silkworm infection model. $^{23}$ Therefore, we assumed the mraZ might related to the $S$. aureus pathogenicity. We first found the hemolysis capability of the mraZ deletion mutant was significantly reduced, which may weaken the ability to induce acute infections such as abscess and pneumoniae. ${ }^{24}$ We evaluated the killing ability of the wide-type strain and the mutants in the G. mellonella infection model, and we found the mraZ deletion mutant was less virulent. Given that $S$. aureus often cause skin and soft tissue infections (SSTIs) in human, mouse skin and soft tissue infection model was performed. Our results indicated that MraZ play an essential role in SSTIs and invasion infections. Moreover, the observed impact of mraZ on neutrophil killing revealed that MraZ contributed to immune evasion, which help explain how MraZ contribute to acute infection. Inasmuch as the success of a $S$. aureus infection depends on the effective evasion of host defenses and neutrophils represent the cornerstone of human innate defenses. ${ }^{25,26}$ The golden pigment of $S$. aureus serves as an antioxidant and is protective in killing by neutrophils, while our results indicating that the immune evasion mediated by MraZ was not associated this mechanism. ${ }^{26}$ Furthermore, our results of the cytokine TNF- $\alpha$ and IL-6 level from mouse bacteremia model were also verified the effect of $m r a Z$ on the immune defense. These findings indicated that MraZ might was a virulence factor of $S$. aureus, and play an important role on the virulence and immune evasion in $S$. aureus infections.

Our RNA-seq analysis suggested that MarZ was a regulator of expression of extensive genes and many of which were related to $S$. aureus infection and immune evasions. It is evident that the initiates and maintains of 
S. aureus infection involves an astounding array of virulence determinants. ${ }^{25}$ The MSCRAMM surface proteins A (clfA) that mediates attachment and invasion of tissue cells was decreased in $\Delta m r a Z .{ }^{17}$ The $\alpha$-hemolysin is an important virulence factor in $S$. aureus infections; it can cause pore formation in a series of target cells and epithelial and endothelial breach to help systemic infection. ${ }^{24}$ We observed that the expression of $\alpha$-hemolysin were decreased in $\Delta m r a Z$. Additionally, PSMs and $\delta$-hemolysin were also markedly decreased in $\Delta m r a Z$; these cytolytic toxins can drive attack and eliminate immune cells such as neutrophils and promote immune evasion. Moreover, after $S$. aureus have been managed to ingest by neutrophils, it has reported have several mechanisms providing resistance to antimicrobial peptides (AMPs) with positively charge. ${ }^{1}$ Among them, the dlt operon can mediate the introduction of positive charge, increase the net charge of the cell surface, and inhibit neutrophil killing. ${ }^{16,27}$ Consistently, our data revealed that the deletion of $m r a Z$ can significantly reduce the expression of dlt. These findings confirmed that mraZ involved in virulence mechanisms of $S$. aureus and further explained that the significant effect of $m r a Z$ on virulence phenotype.

$S$. aureus has evolved a complex regulatory network to control virulence. It has well known that agr quorum-sensing system, some TCS systems saeRS, srrAB and arlRS and the assistant regulator $\operatorname{sar} A .{ }^{18}$ Among them, agr can positively regulate $\alpha$-hemolysin gene (hla), PSM genes (pmsal- pmsa4, psm $\beta 1$ and $p s m \beta 2)$ and $\delta$-hemolysin gene $(h l d) ;{ }^{28}$ sar $A$ can enhances the accumulation of many virulence factors via positive regulation of agr activity. ${ }^{18,29}$ In this study, we found that the deletion of $m r a Z$ resulted in decreasing expressions of $a g r$ and $\operatorname{sar} A$, indicating that the $m r a Z$ can lead to the high expression of many virulence genes by upregulating agr, sarA. This notion is further supported by the reduced production of $a g r-$ dependent virulence factors in the mutant with reduced.

However, there are still some limitations in our study. Most of the phenotypic experiments of the complementary strain have not completely recovered to the level of its parent strain. The probable reason may be associated to the genes that only exists on the plasmid and did not restore in their chromosome. Although we confirmed that mraZ plays an important role in the virulence of $S$. aureus, and regulating some virulence genes and regulators, the regulatory mechanisms of $m r a Z$ in relation to virulence requires further investigated.

\section{Conclusion}

Our work demonstrates the $m r a Z$ plays vital roles in regulating many virulence genes via modulation of agr and
sarA expression in S. aureus. MraZ is an attractive target for anti-virulence therapy of $S$. aureus.

\section{Author Contributions}

FY and YG conceived and drafted the work. BW, JD, YJ designed of the work and analyzed and interpreted of data for the work. QZ, YX, HZ, XW and LR participated in the experimental work and data analysis.FY agreed to be accountable for all aspects of the work in ensuring that questions related to the accuracy or integrity of any part of the work are appropriately investigated and resolved. All authors read and approved the final manuscript.

\section{Funding}

This work was supported by the National Natural Science Foundation of China (81871704).

\section{Disclosure}

The authors report no conflicts of interest in this work.

\section{References}

1. Otto M. Staphylococcus colonization of the skin and antimicrobial peptides. Expert Rev Dermatol. 2010;5(2):183-195. doi:10.1586/ edm.10.6

2. Tong SY, Davis JS, Eichenberger E, Holland TL, Fowler VG. Staphylococcus aureus infections: epidemiology, pathophysiology, clinical manifestations, and management. Clin Microbiol Rev. 2015;28(3):603-661. doi:10.1128/CMR.00134-14

3. Tacconelli E, Tumbarello M, Cauda R. Staphylococcus aureus infections. N Engl J Med. 1998;339(27):2026-2027.

4. Centers for Disease Control and Prevention. Active bacterial core surveillance report, emerging infections program network, methicillin-resistant Staphylococcus aureus. 2014.

5. Dinges MM, Orwin PM, Schlievert PM. Exotoxins of Staphylococcus aureus. Clin Microbiol Rev. 2000;13(1):16-34, table of contents. doi:10.1128/CMR.13.1.16

6. Risley AL, Loughman A, Cywes-Bentley C, Foster TJ, Lee JC. Capsular polysaccharide masks clumping factor A-mediated adherence of Staphylococcus aureus to fibrinogen and platelets. $J$ Infect Dis. 2007;196(6):919-927. doi:10.1086/520932

7. Thammavongsa V, Kim HK, Missiakas D, Schneewind O. Staphylococcal manipulation of host immune responses. Nat Rev Microbiol. 2015;13(9):529-543. doi:10.1038/nrmicro3521

8. Otto M. Staphylococcus aureus toxins. Curr Opin Microbiol. 2014;17:32-37. doi:10.1016/j.mib.2013.11.004

9. Dickey SW, Cheung GYC, Otto M. Different drugs for bad bugs: antivirulence strategies in the age of antibiotic resistance. Nat Rev Drug Discov. 2017;16(7):457-471. doi:10.1038/nrd.2017.23

10. Rasko DA, Sperandio V. Anti-virulence strategies to combat bacteria-mediated disease. Nat Rev Drug Discov. 2010;9 (2):117-128. doi:10.1038/nrd3013

11. Eraso JM, Markillie LM, Mitchell HD, Taylor RC, Orr G, Margolin W. The highly conserved MraZ protein is a transcriptional regulator in Escherichia coli. J Bacteriol. 2014;196(11):2053-2066. doi:10.1128/JB.01370-13 
12. Maeda T, Tanaka Y, Takemoto N, Hamamoto N, Inui M. RNase III mediated cleavage of the coding region of mraZ mRNA is required for efficient cell division in Corynebacterium glutamicum. Mol Microbiol. 2016;99(6):1149-1166. doi:10.1111/mmi.13295

13. Trespidi G, Scoffone VC, Barbieri G, Riccardi G, Rossi ED, Buroni S. Molecular characterization of the Burkholderia cenocepacia dew Operon and FtsZ Interactors as new targets for novel antimicrobial design. Antibiotics. 2020;9(12):841. doi:10.3390/ antibiotics9120841

14. Bae T, Schneewind O. Allelic replacement in Staphylococcus aureus with inducible counter-selection. Plasmid. 2006;55(1):58-63. doi:10.1016/j.plasmid.2005.05.005

15. Montgomery CP, Boyle-Vavra S, Roux A, Ebine K, Sonenshein AL, Daum RS. CodY deletion enhances in vivo virulence of community-associated methicillin-resistant Staphylococcus aureus clone USA300. Infect Immun. 2012;80(7):2382-2389. doi:10.1128/ IAI.06172-11

16. Collins LV, Kristian SA, Weidenmaier C, et al. Staphylococcus aureus strains lacking D-alanine modifications of teichoic acids are highly susceptible to human neutrophil killing and are virulence attenuated in mice. $J$ Infect Dis. 2002;186(2):214-219. doi:10.1086/ 341454

17. Farnsworth CW, Schott EM, Jensen SE, et al. Adaptive upregulation of clumping factor A (ClfA) by Staphylococcus aureus in the obese, type 2 diabetic host mediates increased virulence. Infect Immun. 2017;85(6). doi:10.1128/IAI.01005-16.

18. Jenul C, Horswill AR, Fischetti VA. Regulation of Staphylococcus aureus virulence. Microbiol Spectr. 2019;7(2). doi:10.1128/microbiolspec.GPP3-0031-2018

19. Hua L, Hilliard JJ, Shi Y, et al. Assessment of an anti-alpha-toxin monoclonal antibody for prevention and treatment of Staphylococcus aureus-induced pneumonia. Antimicrob Agents Chemother. 2014;58 (2):1108-1117. doi:10.1128/AAC.02190-13

20. Wang Y, Di H, Chen F, et al. Discovery of benzocycloalkane derivatives efficiently blocking bacterial virulence for the treatment of methicillin-resistant S. aureus (MRSA) infections by targeting diapophytoene desaturase (CrtN). J Med Chem. 2016;59(10):4831-4848. doi:10.1021/acs.jmedchem.6b00122
21. Chan WT, Espinosa M, Yeo CC. Keeping the wolves at bay: antitoxins of prokaryotic type II toxin-antitoxin systems. Front $\mathrm{Mol}$ Biosci. 2016;3:9. doi:10.3389/fmolb.2016.00009

22. Dienemann C, Bøggild A, Winther KS, Gerdes K, Brodersen DE. Crystal structure of the VapBC toxin-antitoxin complex from Shigella flexneri reveals a hetero-octameric DNA-binding assembly. J Mol Biol. 2011;414(5):713-722. doi:10.1016/j.jmb.2011.10.024

23. Kyuma T, Kimura S, Hanada Y, Suzuki T, Sekimizu K, Kaito C. Ribosomal RNA methyltransferases contribute to Staphylococcus aureus virulence. Febs J. 2015;282(13):2570-2584. doi:10.1111/ febs. 13302

24. Vandenesch F, Lina G, Henry T. Staphylococcus aureus hemolysins, bi-component leukocidins, and cytolytic peptides: a redundant arsenal of membrane-damaging virulence factors? Front Cell Infect. 2012;2:12

25. Cheung GYC, Bae JS, Otto M. Pathogenicity and virulence of Staphylococcus aureus. Virulence. 2021;12(1):547-569. doi:10.1080/21505594.2021.1878688

26. Jong NW, Kessel KP, Strijp JA. Immune evasion by Staphylococcus aureus. Microbiol Spectr. 2019;7(2):7-12.

27. Peschel A, Otto M, Jack RW, Kalbacher H, Jung G, Götz F. Inactivation of the dlt Operon inStaphylococcus aureus confers sensitivity to defensins, protegrins, and other antimicrobial peptides J Biol Chem. 1999;274(13):8405-8410. doi:10.1074/jbc.274.13.8405

28. Cheung GY, Wang R, Khan BA, Sturdevant DE, Otto M, Payne SM. Role of the accessory gene regulator agr in community-associated methicillin-resistant Staphylococcus aureus pathogenesis. Infect Immun. 2011;79(5):1927-1935. doi:10.1128/IAI.00046-11

29. Zielinska AK, Beenken KE, Joo HS, et al. Defining the strain-dependent impact of the Staphylococcal accessory regulator (sarA) on the alpha-toxin phenotype of Staphylococcus aureus. J Bacteriol. 2011;193(12):2948-2958. doi:10.1128/JB.01517-10
Infection and Drug Resistance

\section{Publish your work in this journal}

Infection and Drug Resistance is an international, peer-reviewed openaccess journal that focuses on the optimal treatment of infection (bacterial, fungal and viral) and the development and institution of preventive strategies to minimize the development and spread of resistance. The journal is specifically concerned with the epidemiology of

\section{Dovepress}

antibiotic resistance and the mechanisms of resistance development and diffusion in both hospitals and the community. The manuscript management system is completely online and includes a very quick and fair peerreview system, which is all easy to use. Visit http://www.dovepress.com/ testimonials.php to read real quotes from published authors. 